effort to avoid their hospitalisation. In these cases the patients were subsequently transferred directly to an acute psychiatric ward from Brunswick House. With the benefit of experience, patients in a similar position are now less likely to be accommodated. These few patients heavily bias the results and their inclusion (to a certain extent) balances the regression towards the mean argument. There is also a reduction in accident and emergency attendance, which may be a consequence of the acceptability of Brunswick House to those with self-harming behaviours. The absence of serious self-harming behaviour and violence to others while at Brunswick House contrasts with the prevalence of such behaviours on acute wards. This cannot be accounted for solely by admission bias, as many prolific self-harming patients have been accommodated at Brunswick House. Indeed, this observation has led to the development of a self-help group for people with this problem, based at Brunswick House.

Since it opened Brunswick House has been fully occupied, and an increasing number of potential users have not been accommodated. As a result restrictions have been placed on the number of times a person can be accommodated in a year. This has led to many previous residents receiving telephone support from Brunswick House staff. These pressures have resulted in discussions about opening another crisis house in the county. Even though the unit is fully occupied, efforts have been made to attract ethnic minority users who are significantly underrepresented. Our results and the increased demand for accommodation at Brunswick House suggest that the unit provides a valuable service to the people of North Staffordshire and demonstrates an effective partnership between non-statutory and statutory services.

\section{Declaration of interest}

None.

original papers

\section{References}

BECK, A., CROUDACE, T. J., SINGH, S., et HAYCOX, A., UNSWORTH, L., ALLEN, al (1997) The Nottingham Acute Bed K., et al (1999) The North Staffordshire Study: alternatives to acute psychiatric Community Beds Study. Longitudinal care. British Journal of Psychiatry, 170, evaluation of beds attached to 247-252

BOARDMAN, A. P., HODGSON, R. E., LEWIS, M., et al (1999) The North Staffordshire Community Beds Study. Longitudinal evaluation of beds attached to community mental health centres. I. Methods, outcome and patient satisfaction. British Journal of Psychiatry, 175, 70-78.

BOND, G. R., WITHERIDGE,T. F., WASMER, D., et al (1989) A comparison of two crisis housing alternatives to psychiatric

hospitalisation. Hospital and Community Psychiatry, 40, 177-183.

DEPARTMENT OF HEALTH (1996) The Spectrum of Care: Local Services for People with Mental Health Problems. London: HMSO.

FLANNIGAN, C. B., GLOVER, G. R. FEENEY, S. T., et al (1994) Inner London collaborative audit of admissions in two health districts. 1: Introduction, methods and preliminary findings. British Journal of Psychiatry, 165, 734-742.

*Richard Hodgson Senior Lecturer in Psychiatry, Lyme Brook Mental Health Centre, Bradwell Hospital Site, Talke Road, Stoke-on-Trent ST5 7TL, Darren Carr Senior House Officer, Lyme Brook Mental Health Centre, Stoke-on-Trent, Lynne Wealleans Manager, Brunswick House, Stoke-on-Trent

\title{
Induction agents in electroconvulsive therapy: a comparison of methohexitone and propofol
}

\section{AIMS AND METHOD}

To compare methohexitone and propofol in electroconvulsive therapy (ECT). A retrospective within-subject comparison was made of the use of these drugs in separate courses of bilateral ECT in one clinic over 10 years. Patients taking mood stabilising or anti-epileptic drugs were excluded. The initial seizure threshold and seizure duration were of particular interest.

RESULTS

The median initial seizure thresholds were identical $(75 \mathrm{mC})$. The median initial seizure duration with threshold stimulation was $25 \%$ shorter with propofol ( 21 v. 28 s). The median total numbers of treatments in the courses were identical (eight treatments).

\section{CLINICAL IMPLICATIONS}

The shorter seizure duration observed with propofol was not associated with a commensurate rise in the initial seizure threshold. The shorter seizure duration may therefore have no effect on the therapeutic efficacy of treatment.
Methohexitone ceased to be available commercially in the UK in 1999. At that time it was the drug of choice for the induction of anaesthesia in electroconvulsive therapy (ECT) (Special Committee on ECT, 1995). Many 
anaesthetists chose propofol as its replacement because it is a non-barbiturate drug that is metabolised rapidly

original papers

and causes less haemodynamic change than methohexitone. Some psychiatrists were concerned about the use of propofol because they observed that its use was associated with shorter seizures. This raised the concern that induction with propofol might compromise the therapeutic efficacy of ECT. Randomised, controlled comparisons of induction with propofol and methohexitone have not supported this concern: see Martin et al (1998) for a review of the literature. Nevertheless, some practitioners remain concerned about the use of propofol and there continue to be anecdotal reports of difficulties in the induction of seizures that are attributed to the use of propofol. Seizure duration itself has never been shown to be related to the therapeutic efficacy of ECT (Scott, 1989), but if the shorter seizures observed with propofol were associated with a rise in seizure threshold this might be a cause for concern. The extent to which the electrical dose exceeds the seizure threshold has been shown to be related to the therapeutic efficacy of ECT in the treatment of depressive illness (American Psychiatric Association, 2001). No study of the initial seizure threshold in ECT has ever compared induction with methohexitone and propofol. A prospective study is now impossible, and this prompted the study reported here.

\section{Method}

The index card records at the ECT clinic at the Royal Edinburgh Hospital contain information on treatments from January 1991 onwards. These were searched by hand up to and including the records for December 2000 to identify patients who had undergone both induction with methohexitone and induction with propofol in separate courses of ECT. Methohexitone became unavailable in July 1999. The further inclusion criterion was the initial prescription of a bilateral electrode placement. Exclusion criteria were the intake of an anti-epileptic or mood stabilising drug (excluding lithium carbonate) and ECT in the previous 6 months. Twenty patients satisfied the initial inclusion and exclusion criteria.

Treatment was always given using a machine from the Ectron $5 \mathrm{~A}$ series and therefore the characteristics of electrical stimulation were standard throughout the study. Empirical measurement of the initial seizure threshold was carried out routinely at the clinic, and the

Table 1. A within-subject comparison of methohexitone and propofol induction of electroconvulsive therapy $(n=13)$

\begin{tabular}{|lcc} 
& \multicolumn{2}{c}{ Induction agent } \\
\cline { 2 - 3 } & $\begin{array}{c}\text { Methohexitone } \\
\text { Treatment detail }\end{array}$ & $\begin{array}{c}\text { Propofol } \\
\text { median (range) }\end{array}$ \\
median (range)
\end{tabular}

treatment protocol has been described before (Special Committee on ECT, 1995). Four patients had to be excluded from the study because the treatment protocol was not followed sufficiently closely to give an accurate measurement of initial seizure threshold at one of the courses. Two other patients had to be excluded because they were subsequently prescribed an anti-epileptic or mood stabilising drug. One patient had to be excluded because she was switched to a unilateral electrode placement. Seizure duration was recorded routinely and measured from the end of electrical stimulation to the end of generalised convulsive muscular activity.

Neither initial seizure threshold nor initial seizure duration was normally distributed, and non-parametric statistics have been used throughout.

\section{Results}

The sample consisted of 13 patients, 6 men with a median age of 38 (range 27-67) years, and 7 women with a median age of 51 (range 30-79) years. About half of the people in the sample $(n=6)$ suffered from recurrent depressive disorder in which the treated episodes were severe. Five of the patients suffered from a manicdepressive psychosis and the treatments were prescribed for severe depressive episodes; one patient who suffered from a manic-depressive psychosis was treated each time for mania. One patient suffered from a depressive episode in a schizoaffective disorder. The median length of time between treatment courses was 25 (range 10-71) months. Table 1 shows that the median initial seizure thresholds were identical, $75 \mathrm{mC}$ (Wilcoxon matchedpairs signed ranks test, $n=9, t=19$, critical value 6 , not significant). The median initial seizure duration with threshold stimulation was $25 \%$ shorter when propofol was used ( $n=12, t=8$, critical value 10 , two-tailed $P=0.02$ )

Two patients decided to stop one of the courses of ECT prematurely; one man with hypomania withdrew consent and one woman with depression went on holiday. The total number of treatments was therefore based on the 11 patients who completed the prescribed courses of treatment. The median total numbers of treatments in the course were also identical $(n=8, t=14$, critical value 4 , not significant).

\section{Discussion}

This was not the first study to confirm the impressions of practitioners that seizure duration is shorter when propofol is used as the induction agent (see Martin et al, 1998). The novel finding from this study was that this can occur without any commensurate increase in the initial seizure threshold in bilateral ECT. The median initial seizure threshold with propofol induction was identical to the median value found in an earlier study of 100 depressed patients treated with the same ECT machine in whom methohexitone was the induction agent (Dykes \& Scott, 1998). The findings add to the evidence that seizure duration and seizure threshold are only modestly 
correlated in ECT (Scott \& Boddy, 2000). That the shorter seizures were not associated with a higher seizure threshold makes it less likely that propofol compromises the therapeutic efficacy of ECT. The lack of a difference in the total number of prescribed treatments lends support to this.

Methohexitone is still not available in our clinic and a retrospective comparison is all that is feasible. The strengths of this study are that it was conducted on a representative sample from one clinic over a defined period, and had a within-subject design. Its major limitation is that it lacked the statistical power to conclude confidently that the two induction agents were equivalent in their effect on the seizure threshold. The study would have been strengthened if cerebral seizure activity had been measured directly by electroencephalography, but this was not available routinely in the clinic. There are other reasons to be cautious in the interpretation of the findings. It was beyond the scope of the study to assess the effects of concurrent psychotropic drug treatment, or the effect of increasing age over the period of the study (age is positively correlated with the initial seizure threshold). Measures of clinical outcome normally seen in treatment studies were not available.

The clinical implication is that the study has added to the evidence that the shorter seizures observed after induction with propofol need not compromise the therapeutic efficacy of ECT.

\section{Acknowledgements}

We thank Staff Nurse Rena Good of the ECT clinic at the Royal Edinburgh Hospital for her help with the study. We also thank Alex Celini, Research Assistant for the Royal College of Psychiatrists' Special Committee on ECT, for preparing the manuscript.

\section{Declaration of interest}

None.

\section{References}

AMERICAN PSYCHIATRIC ASSOCIATION (2001) The Practice of ElectroconvulsiveTherapy: Recommendations for Treatment, Training, and Privileging (2nd edn) Washington, DC: APA.

DYKES, S. \& SCOTT, A. I. F. (1998) Seizure threshold in bilateral electroconvulsive therapy. Psychiatric Bulletin, 22, 298-299.

MARTIN, B. A., COOPER, R. N. \& PARIKH, S. V. (1998) Propofol anaesthesia, seizure duration and ECT:

a case report and literature review. Journal of ECT, 14, 99-108.

SCOTT, A. I. F. (1989) Which depressed patients will respond to electroconvulsive therapy? British Journal of Psychiatry, 154, 8-17.

- \& BODDY, H. (2000) The effect of repeated bilateral ECT on the seizure threshold. Journal of ECT, 16, 244-251.

SPECIAL COMMITTEE ON ECT (1995) The ECTHandbook. London: Gaskell.

*Allan Scott Consultant Psychiatrist and Honorary Senior Lecturer, c/0 Research/Administration Assistant, Special Committee on ECT,The Royal College of Psychiatrists, Scottish Division, 9 Queen St, Edinburgh EH2 1JQ, UK, Harold Boddy Senior House Officer in Psychiatry, Andrew Duncan Clinic, Royal Edinburgh Hospital, Morningside Terrace, Edinburgh EH10 5HF 